\title{
Sprinter joint point identification model of biomechanics
}

\author{
Ziwen Wang ${ }^{1, a}$, Qiaohui Wang ${ }^{2}$, Kunling Qin ${ }^{3}$, Qian Yang ${ }^{1}$ and Bing Zhang ${ }^{1, b}$ \\ ${ }^{1}$ Institute of Physical Education, Huanggang Normal University, Huangzhou 438000, China \\ ${ }^{2}$ Macaomiao Primary School, Tuanfeng, Hubei, China \\ ${ }^{3}$ Yidu Gaobazhou Middle School, Yidu 443300, China
}

\begin{abstract}
This article USES the automatic identification of human body joint point in the process of sports science algorithm, as a key point to discuss, in order to more realistic three-dimensional reconstruction basis of science. Through the body's automatic identification of the key software implementation process research, and in this paper, the image processing model to reconstruct the human body model according to the proportion principle in the late. Using camera measurement instruments and scientific measurement method, provides effective scientific data collection. Research results, to determine the link of 15 and 21 key points of the body, and gives the mathematical model of each joint point determined to establish the discriminate model of special circumstances, are visible side joint point enter human body contour line, invisible side joint point of shade and hands on hip to determine the influence of processing method.
\end{abstract}

\section{Introduction}

Sports scientific advancement relies on modern science and technological development, and gets profit from science and technological development, especially after electronic measurement technology and computer image processing technology developing. The paper takes running's athletes as research objects, for its contour extraction algorithm and articulation point extraction algorithm, it makes analysis, and in the hope of exploring more effective human movement articulation point automatic recognition system, and provides basis for Chinese sports development [1-3].

The paper based on former researches' running process human each articulation point extraction system; it makes analysis, in the hope of letting sports analysis to be more scientific and effective by the paper researches.

\section{Athletes human model's each articulation point defining and software recognition process}

For indicators than can monitor and these indicators feedback information, adjust athletes training contents is basis of current scientific training, that is to say, is a kind of sports training model that adapts to social and science and technological development. In current sports training, in general, it adopts movement videos analysis method to extract monitoring indicators, and mines training process shortcomings from monitoring indicators changing features, in the hope of providing basis for more scientific

a,b Corresponding author: ${ }^{\mathrm{a} T y-w z w} @$ hgnu.edu.cn; and reasonable sports training improvements, but applying movement video analysis method has relative complex implementation process, and it will appear certain deviations in operational implementation, which can let numerous scholars to continue to pursue on movement identification and indicators data mining simplified implementation that can monitor. In order to explore more simple movement recognition algorithm, the paper takes running process athletes as research objects, researches on human model's articulation point extraction and recognition process, in the hope of making contributions to simplified movement recognition $[4,5]$.

To sum up, the paper research target is designing simplified human movement recognition system, and simplified human recognition system designing starting point is applying model method to monitor movement articulation point movement features. Therefore, the chapter divides two sections to make statement and illustration on athletes each articulation point software recognition flow and athlete human model's each articulation point defining, in the hope of providing basis for simplified human movement recognition system algorithm designing [6].

\subsection{Athlete each articulation point software recognition flow}

The paper adopts windows operating system platform [7], and runs sprint human articulation point automatic recognition software system in the platform. Human articulation point automatic recognition software system is composed Figure 1 showed video converted chattering free digital image, input image, human articulation point 
automatic recognition, manual modifying specific points, output data, exit and help seven main modules.

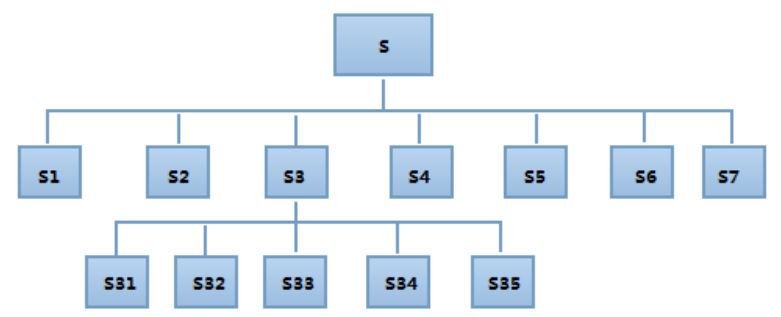

Figure 1. Software system structure schematic diagram.

When apply the system software to do human articulation point automatic recognition, after inputting images, the system can automatic enter into human articulation point automatic recognition phase without any manual operating, software will recognize every image human articulation point, recognition process is firstly extracting human contour, and then according to human model, extracting skeleton bar chart on contour, finally targeted at skeleton connection points, which is extracting data of human articulation points changing status, applying menu's data output and printing basic data report into data extracted data, and getting each articulation point corresponding coordinate, recognition effect graph is as Figure 2 shows.

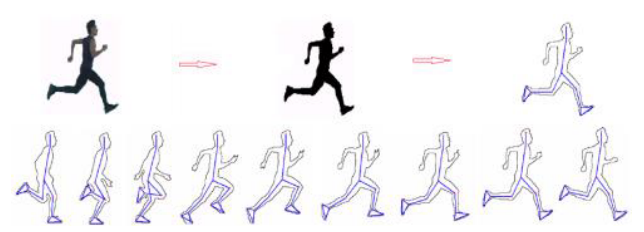

Figure 2. Human articulation point recognition process and recognition efficiency graph.

\subsection{Athlete human model's each articulation point defining}

In software image post processing procedures, it should restore human initial size according to certain proportion, in order to research objects human articulation point tracking, it needs to firstly define human tracking pint, human tracking point and skeleton located postures measurement status is as Figure 3 shows.

In order to achieve the purpose of simplifying calculation amount and recognition effectiveness, it should carry on tracking during movement process targeted at feature points nearby regions [8]. Sprint athlete movement has complex, his body each link doesn't make constant movement in movement process, traditional predicting next moment sports status only relies on previous moment sports status cannot correctly proceed, in addition, in recognition process, it will appear status that visible lateral articulation point enters into human contour line and invisible lateral articulation point sheltered, hand definite impacts on hip joints as well as other specialties, let recognition process to lead to interruption, therefore the paper firstly utilizes obtained human contour line highest point A、lowest point B to define moving body basic positioning and get basic proportional length $L_{H I}$, and then according to this, define human external contour points $\mathrm{C}, \mathrm{D}, \mathrm{E}, \mathrm{F}, \mathrm{G}, \mathrm{H}$ and finally according to already obtained feature points, combines with sprinting rules to define human each articulation point regional tracking, for each articulation point specialties and each time phase specialties, for different time phases each articulation point, adopts different tracking region center and radius, and makes adaptive adjustment according to motions coherences.
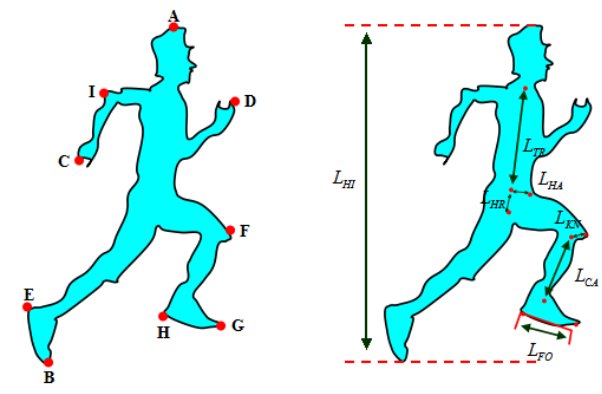

Figure 3. Runner human contour region tracking and proportional length defining.

Sprint is a periodic movement event, for the convenience purpose, it can regard the event as being composed of double steps one by one, and every double step is composed of two single steps, for every single step, it can be divided into supporting time phase, soaring one time phase and soaring two time phases totally three time phases, three time phases separation are foot landing instant, foot out of ground instant, lowest point changing from one foot to another foot instant as well as foot re-landing instant.

Single step three time phases' four time features separation's definition in corresponding images magnitudes are as following shows:

Out of ground instant corresponding magnitude: human contour lowest point vertical displacement occurs obvious change, the change already goes beyond error fluctuation range instant corresponding magnitude

Landing instant corresponding magnitude: In case that human contour lowest point's vertical displacement changes from variation to static, one fluctuation range time corresponding magnitude.

Lowest point transforms from left foot to right foot corresponding magnitude: human contour lowest point horizontal displacement occurs obvious changes; the change surpasses set range instant corresponding magnitude.

Data distribution features are as Figure 4 show.

In Figure 4, corresponding magnitude expressive definitions are as following show:

1) $\mathrm{n} 1$ represents the first time landing instant corresponding magnitude.

2) n2 represents first time leaving instant corresponding magnitude.

3) $n 3$ represents the first time lowest point left foot to right foot transformation corresponding magnitude.

4) n4 represents the second time landing instant corresponding magnitude.

5) n5 represents the second time lowest point left foot 
to right foot transformation corresponding magnitude.

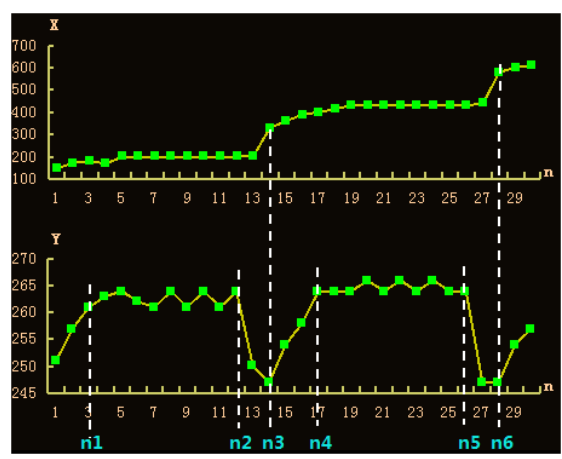

Figure 4. Contour lowest point coordinate value change trend with magnitudes.

\section{Sprint athlete human articulation point automatic recognition mathematical modeling}

\subsection{Research objects and measurement method}

Research objects: The paper takes second grade 10 sprint athletes as research objects, clothes is dark skintight outfits and dark shoes and stockings without attaching any mark points in body, background color is light fixed wall.

Measurement method: Apply two-dimensional fixed video camera measurement principle; video camera shooting frequency is $50 \mathrm{HZ}$ installation position is as Figure 5 shows.

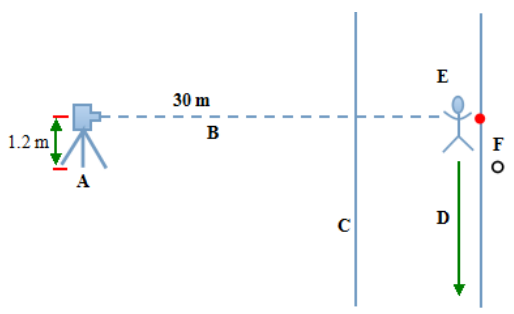

Figure 5. Two-dimensional fixed shooting schematic diagram.

Figure 5 each symbol description is as following show:

A represents video camera

B represents principal optic axis

C represents track

D represents movement direction

E represents sprint athlete

F represents origin

Video camera placed position lets principal optic axis to be vertical to track, video camera nose and athletes distance is $30 \mathrm{~m}$, video camera height is $1.2 \mathrm{~m}$, range of video camera field of view is $10 \mathrm{~m}$, research objects 10 athletes run successively and every athlete sprint for six times, his average speed is controlled around $7 \mathrm{~m} / \mathrm{s}$.

\subsection{Human articulation joint defining and its mathematical model}

According to Hanavan mathematical model, it is clear that human can be expressed as mathematical model that is composed of 15 links by spherical hinge connections, the model classifies human into 15 links as following shows:

1) Head -start from top of head to the seventh cervical vertebra, one piece.

2) Upper trunk-Start from the seventh cervical vertebra to inferior margin of breastbone, one piece.

3) Lower trunk-Start from inferior margin of breastbone to greater trochanter, one piece.

4) Upper arm-Start from shoulder joint center to elbow joint center, two pieces.

5) Forearm-Start from elbow joint center to wrist joint center, two pieces.

6) Hand-Start from wrist joint center to metatarsophalangeal joints center, two pieces.

7) Thigh-Start from hip joint center to knee joint center, two pieces.

8) Shank-Start from knee joint center to ankle joint, two pieces.

9) Foot-Start from calcaneal tuber to toe, two pieces.

In the time that before and after athlete pedaling backward and leaving instant and landing leg will land soon, knee joint angle is larger, knee joint nearby contour line inflection point features are not obvious, it cannot apply inflection point midpoint to define knee joint, therefore take ankle joint $A\left(X_{A}, Y_{A}\right)$ as the center of a circle, and take shank length $L_{C A}$ as radius to draw round, intersection points with knee joint nearby contour line are $B, C$ the two intersection points center is supposed to be knee joint point, as Figure 6 shows.

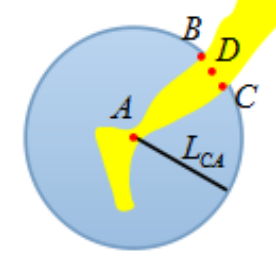

Figure 6. Knee joint point.

As Figure 7 showed knee joint point $D$ coordinate solution way is as formula (1) shows:

$$
\left\{\begin{array}{l}
\sqrt{\left(X_{A}-X_{B}\right)^{2}+\left(Y_{A}-Y_{B}\right)^{2}}=\sqrt{\left(X_{A}-X_{B}\right)^{2}+\left(Y_{A}-Y_{B}\right)^{2}}=L_{C A}(1) \\
X_{D}=\frac{1}{2}\left(X_{B}+X_{C}\right), Y_{D}=\frac{1}{2}\left(Y_{B}+Y_{C}\right)
\end{array}\right.
$$

Similarly, apply link length method, it can solve hip joint coordinate, top head coordinate and inferior margin of breastbone coordinate.

Apply shortest distance midpoint method to define the seventh cervical vertebra point, firstly it needs to define two contours lines shortest distance two points $A\left(X_{A}, Y_{A}\right)$ and $B\left(X_{B}, Y_{B}\right)$, and take $C\left(X_{C}, Y_{C}\right)$ as the seventh cervical vertebra point, as Figure 7 shows.

Simultaneous formula (2), it can get the seventh cervical vertebra point coordinate $C\left(X_{C}, Y_{C}\right)$.

$$
\left[X_{C}=\frac{1}{2}\left(X_{A}+X_{B}\right), Y_{C}=\frac{1}{2}\left(Y_{A}+Y_{B}\right)\right]
$$




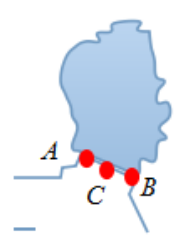

Figure 7. The seventh cervical vertebra point.

Apply link length method to define knee joint point, the method application evidence is in sprinting process, moving body each link vertical axis is always vertical to video camera principal optic axis, therefore in image each link length is supposed to remain unchanged, apply fixed link length to define articulation point coordinate.

Apply vertical link tangent line midpoint method, it can solve shoulder joint coordinate, it needs to firstly define upper arm two ends contour line's feature points $A\left(X_{A}, Y_{A}\right)$ and $B\left(X_{B}, Y_{B}\right)$, in line segment $A B$ distance $A$ point as $\frac{1}{5} A B$ area, take point $F\left(X_{F}, Y_{F}\right)$ to make vertical line segment $A B$ vertical line, the straight line and contour line intersect in $C\left(X_{C}, Y_{C}\right)$ and $D\left(X_{D}, Y_{D}\right)$, take two intersection points midpoint $E$ as shoulder joint point, as Figure 8 shows.

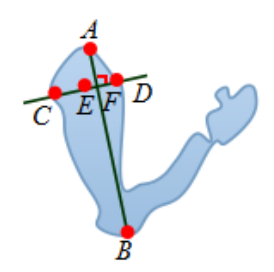

Figure 8. Shoulder joint point.

As Figure 8shows, simultaneous equation set formula (3), it can get point $E$ coordinate:

$$
\left\{\begin{array}{l}
X_{F}=X_{A}+\frac{1}{5}\left(X_{B}-X_{A}\right), Y_{F}=Y_{A}+\frac{1}{5}\left(Y_{B}-Y_{A}\right) \\
\frac{\left|Y_{C}-Y_{F}\right|}{\left|X_{F}-X_{C}\right|}=\frac{\left|X_{B}-X_{A}\right|}{\left|Y_{B}-Y_{A}\right|}=\frac{\left|Y_{D}-Y_{F}\right|}{\left|X_{F}-X_{D}\right|} \\
X_{E}=\frac{1}{2}\left(X_{C}+X_{D}\right), Y_{E}=\frac{1}{2}\left(Y_{C}+Y_{D}\right)
\end{array}\right.
$$

Similarly apply vertical link tangent line midpoint method; it can solve wrist joint and ankle joint coordinate.

\section{Conclusion}

The paper firstly analyzes human articulation point automatic recognition software implementation flow, and states image post processing human model's model principle that reconstructs according to proportions. It studies on video camera measurement apparatus and scientific measurement method, which provides basis for effective and scientific data collection. It defines human body 15 links and 21 articulation points, and provides each articulation point defined mathematical model. It analyzes image overlapping and other specific cases, establishes special cases discriminant model, provides visible lateral articulation point entering into human contour line, invisible lateral articulation point sheltering and hand definite impacts on hip joint processing method.

\section{References}

1. X.J. Zhou, Z.C. Mao, Sports \& Science. 32, 103-106 (2011)

2. Z.Y. Lin, Sports Sciences Researches. 9, 44-49 (2005).

3. D. H. Fu, P. Z. Zhang, X.L. Meng, China Sport Science and Technology. 42, 33-37 (2006).

4. B. Bi, Sports \& Science. 28, 68-70, 67 (2007).

5. K. Liu, Bulletin of Sport Science \& Technology. 16, 18-20 (2008).

6. G.S. Zhao, X.J. Han, J.S. Chen, L.L. Xie, Y.J. Suo, X.H. Hu, Bulletin of Sport Science \& Technology. 19, 10-12 (2011).

7. Y. Lang, Z.L. Liu, H.Y. Liao, Cluster Computing-The Journal of Networks Software Tools and Applications. 18, SI, 507-516 (2015).

8. R. Zhou, J. Teng, Journal of Coastal Research. 73, 600-605 (2015). 